\title{
Efecto de dos sistemas de cosecha de la caña de azúcar sobre la calidad del suelo en Cantón Milagro, Ecuador
}

\author{
Effect of two sugarcane harvest systems on soil quality in Canton of Milagro, Ecuador
}

\author{
Yoansy García ${ }^{1 *}$, Armando Vega ${ }^{1}$ y Rossana Castro ${ }^{1}$
}

RESUMEN

Con el objetivo de evaluar el efecto de las tecnologías de cosecha verde y cosecha quemada en el cultivo de caña de azúcar, Saccharum spp. (Poaceae), sobre la calidad del suelo, se hizo una investigación en los predios de la Universidad Agraria del Ecuador, Cantón Milagro. Se evaluaron variables químicas, físicas y biológicas de los suelos. Se pudo observar que la quema no afectó significativamente la mayoría de las propiedades químicas, y que las más importantes fueron la disminución y bloqueo (antagonismo) de K, y el detrimento de los contenidos de materia orgánica. Esto conllevó a un deterioro de la estructura y a una disminución significativa de la actividad biológica, lo que a su vez provoca una reducción de la capacidad productiva de los suelos. La cosecha de caña mediante la quema constituye un elemento negativo que contribuye a la degradación del medio edáfico.

PALABRAS CLAVE

Saccharum spp., caña quemada, suelo

\begin{abstract}
With the objective to evaluate the effect of green and burned harvest technologies in the cultivation of sugar cane, Saccharum spp. (Poaceae), on soil quality, an investigation was made in the farms of the Agrarian University of Ecuador, Cantón Milagro. Chemical, physical and biological soil were evaluated. It was observed that the burning does not significantly affect majority of the chemical properties, the most important variables were decrease and blockage (antagonism) of K, with detriment of organic matter content which led to a structural deterioration and a significant decrease of biological activity which causes a reduction in the productive capacity of soils. The harvesting of sugarcane through burning constitutes a negative element that contributes to the degradation of the soil environment.
\end{abstract}

KEYWORDS

Saccharum spp., sugar cane, burned sugar cane, soil

${ }^{1}$ Laboratorio de Suelos, Facultad de Ciencias Agrarias, Universidad Agraria del Ecuador. Guayaquil, Ecuador.

* Autor para correspondencia. Avenida 25 de julio y Pío Jaramillo. 090104 Guayaquil, Ecuador.

Correo electrónico: yogarcia@uagraria.edu.ec

Recibido: 13 de abril de 2018.

Aceptado: 2 de julio de 2018. 


\section{INTRODUCCIÓN}

La caña de azúcar, Saccharum officinarum L. (Poaceae), es un cultivo de gran importancia a nivel mundial, ya que de ella se obtienen diversos productos (azúcar, etanol, y otros derivados). Esto la hace atractiva a la hora de su establecimiento, además de que posee una elevada plasticidad agroecológica que le permite ser utilizada en diversos tipos de suelos y climas.

En Ecuador, en 2015, habían plantadas 74,000 ha de caña de azúcar, de las cuales se cosecharon 54,000. Debido a diferentes factores climáticos adversos para la cosecha mecanizada (excesiva lluvia y prolongación del periodo lluvioso) y elevados rendimientos, la cosecha se vio afectada, con lo que quedaron rezagadas $20 \mathrm{mil} \mathrm{ha.} \mathrm{A} \mathrm{pesar} \mathrm{de} \mathrm{este} \mathrm{inconveniente,}$ la producción de caña y de azúcar fue similar a la del año anterior (CINCAE 2017).

Entre los problemas asociados a la implementación del cultivo de la caña de azúcar, se encuentra el relativamente difícil manejo de las plantaciones, entre otras cosas, por su porte alto y gran cantidad de follaje. Estos aspectos predisponen la invasión de diversos animales y plantas que pueden ser perjudiciales para el hombre, especialmente durante la cosecha manual. También el exceso de follaje incrementa la entrada de materias extrañas a la industria, lo que dificulta y hace menos eficiente el proceso industrial de extracción de azúcar. Por todo ello, se prefiere quemar la caña de azúcar, lo que trae consigo una afectación importante al medio ambiente (Ortiz et al. 2012).

La quema de la caña de azúcar, asociada al deterioro del ambiente, es atractiva desde el punto de vista práctico y en cierto sentido económico para los propietarios, e indistintamente se emplea en la cosecha manual como mecanizada. La cosecha manual ofrece mayor protección al cortero, al eliminar el peligro de animales como serpientes, ratas y avispas; por su parte, la cosecha mecanizada disminuye residuos para la industria y mejora la productividad. También se mejora la eficiencia de las cosechadoras y se facilitan las labores de postcosecha (Pongpat et al. 2017).

La quema de la caña de azúcar ocasiona problemas serios a la salud de las personas, principalmente de tipo respiratorios, en especial en niños y ancianos (Arbex et al. 2007). Además, provoca daños medioambientales porque deteriora las propiedades físicas, químicas y biológicas de los suelos. Algunos países como Brasil están transitando de la cosecha quemada hacia la verde; sin embargo, Souza et al. (2012) señalan que, para la materia orgánica del suelo, tiene más impacto cosechar manualmente la caña quemada, ya que la verde y mecanizada ocasiona menor desarrollo de las raíces y conlleva a la reducción de la incorporación de la materia orgánica del suelo debido al alto grado de compactación que produce la maquinaria pesada. Sin embargo, Chaves y Bermúdez (2006) aseveran que la mayoría de los efectos negativos de la quema no tienen soporte o investigación rigurosa que los avale, lo que origina que se especule sobre los daños que ocasiona este tipo de cosecha.

En Ecuador, la forma predominante de cosecha de la caña de azúcar es la quema, debido fundamentalmente a una eficiente estructura que permite al productor hacer llegar la materia prima al ingenio azucarero rápidamente, con lo que se evita el desdoblamiento de la sacarosa, además de los otros beneficios que se mencionaron anteriormente. Por lo anterior, esta investigación se realizó con la finalidad de contribuir al incremento de información que permita conocer mejor el efecto de estas dos tecnologías de manejo en la cosecha de caña de azúcar sobre el medio edáfico, mediante el uso de indicadores de la calidad del suelo.

\section{MATERIALES Y MÉTODOS}

\section{Descripción del sitio de evaluación}

La investigación se realizó en predios de la Universidad Agraria del Ecuador, sede Milagro, perteneciente al Cantón Milagro, de la provincia del Guayas (Coordenadas UTM: 17 M $658226.66 \mathrm{~m} \mathrm{E}$ $9764626.23 \mathrm{~m} \mathrm{~S}$ ). El tipo de suelo fue Vertisol (USDA 2014).

\section{Muestreo y técnicas empleadas}

La variedad de caña de azúcar utilizada fue la CC85-92, de origen colombiano. Se evaluaron dos tratamientos: a) caña quemada y b) caña verde; ambos fueron cosechados de manera manual. El diseño estadístico fue completamente al azar con tres réplicas. Las parcelas estuvieron conformadas por 6 surcos de $8 \mathrm{~m}$ de largo y $1.5 \mathrm{~m}$ entre surcos $(8 \times 9)$; el tamaño de la unidad experimental fue de $72 \mathrm{~m}^{2}$. Se realizó un muestreo antes de la cosecha de la caña, durante el cual fueron tomadas tres muestras de suelo compuestas por tres submuestras para cada propiedad evaluada (física, química y biológica), ya que requerían análisis, procesamiento y almacenamiento diferente, lo que arrojó un total de 18 muestras compuestas. Después de la quema se realizó el mismo procedimiento una 
vez por mes durante tres meses, lo que originó un total de 72 muestras compuestas.

\section{Variables analizadas}

Humedad de Campo. La humedad de campo se determinó por el método gravimétrico.

Actividad biológica. La actividad biológica se realizó mediante la estimación de la respiración de suelo (cuantificación de CO²) (Isermeyer 1952). La estimación del $\mathrm{CO}^{2}$ producido por los microorganismos del suelo se obtuvo de la incubación del suelo en un ambiente anaeróbico a $30{ }^{\circ} \mathrm{C}$ y el suelo a máxima retención hídrica. $\mathrm{El} \mathrm{CO}^{2}$ fue atrapado en una disolución de hidróxido de sodio ( $\mathrm{NaOH} 0,5 \mathrm{~N}$ ), que se valoró posteriormente con ácido clorhídrico $(\mathrm{HCl} 0.5 \mathrm{~N})$.

Agregados estables. Se empleó el método de Pieri (1995), el cual requiere los datos obtenidos de textura (limo y arcilla) más el porcentaje de materia orgánica. Se realizó mediante la siguiente formula:

\section{Indice Estructural o agregados estables $=$ MO/C de Arcilla $+C$ de Limo*100}

\section{Análisis estadístico}

La comparación de medias se realizó mediante la prueba $t$-Student $(p=0.05)$. El software estadístico utilizado fue SPSS versión 23 (IBM Corp. Released 2014).

\section{Análisis multivariado}

Para evaluar las relaciones entre las variables biológicas, físicas y químicas, se realizó el análisis de CoInercia (COIA). Se realizaron dos matrices: una con los datos biológicos y físicos por sitio de muestreo y otra con los valores de las propiedades químicas del suelo determinadas; estos últimos datos se estandarizaron dado que poseen distinta magnitud. Estas matrices se conectaron a través del análisis del COIA. Para determinar la significación de los valores de CoInercia, se realizó la prueba de Monte Carlo. Se utilizó el paquete ADE-4 versión 1.7-8 (Dray y Dufour 2007), incluido en el software RStudio 3.4.2. (R Development Core Team, 2017).

\section{Análisis químicos}

Los análisis químicos se realizaron en el Laboratorio de Suelos, Tejidos Vegetales y Aguas, de la Estación
Experimental del Litoral Sur, acreditado por el Servicio de Acreditación Ecuatoriano. El detalle de la metodología utilizada se muestra en el cuadro 1.

Cuadro 1. Técnicas empleadas en el análisis químico de suelo.

\begin{tabular}{|c|c|c|}
\hline DETERMINACIÓN & Metodología & EXTRACTANTE \\
\hline $\begin{array}{l}\text { Materia orgánica } \\
\text { del suelo }\end{array}$ & Walkey Black & Dicromato de K \\
\hline $\mathrm{NH} 4, \mathrm{P}$ & Colorimetría & $\begin{array}{l}\text { Olsen } \\
\text { modificado } \mathrm{pH} \\
8.5\end{array}$ \\
\hline $\begin{array}{l}\mathrm{K}, \mathrm{Ca}, \mathrm{Mg}, \mathrm{Zn}, \\
\mathrm{Cu}, \mathrm{Fe}, \mathrm{Mn}\end{array}$ & $\begin{array}{l}\text { Absorción } \\
\text { atómica }\end{array}$ & \\
\hline S & Turbidimetría & Fosfato de Ca \\
\hline B & Colorimetría & Monobásico \\
\hline $\mathrm{pH}$ & Potenciométrica & $\begin{array}{l}\text { Suelo:agua } \\
(1: 2.5)\end{array}$ \\
\hline
\end{tabular}

\section{RESULTADOS Y DISCUSIÓN}

\section{Incidencia de las tecnologías de cosecha verde y quemada sobre las propiedades del suelo}

En el cuadro 2 se visualiza que las condiciones del terreno al inicio del experimento mantenían propiedades muy similares, lo que garantizó la homogeneidad de la investigación en este aspecto y trazar una línea base que permitió realizar las comparaciones de la incidencia de los factores estudiados. En los cuadros 3, 4 y 5, se muestran los resultados de los elementos determinados en los tratamientos durante el periodo evaluado, lo que permite inferir las tendencias de los mismos.

Se puede observar que el $\mathrm{pH}$ tuvo una ligera disminución estable en caña de azúcar quemada durante los meses evaluados, lo que coincide con lo reportado por Souza et al. (2012), quienes manifiestan que el $\mathrm{pH}$ disminuye por la quema de la caña de azúcar. Contrario a esto, Mendoza et al. (2000) manifiestan que el $\mathrm{pH}$ puede llegar a subir en la caña quemada por la descarboxilación de los ácidos orgánicos, los cuales pueden llegar a consumir $\mathrm{H}+$. Esta disminución se produjo también en la primera etapa en la caña cosechada verde pero no se mantuvo estable posteriormente.

En cuanto a la materia orgánica (MO), hubo una disminución de 0.5 unidades (2.7 a 2.2) en la caña 
Cuadro 2. Resultados analíticos de caña de azúcar antes de la cosecha bajo dos sistemas de producción en Cantón Milagro, Ecuador.

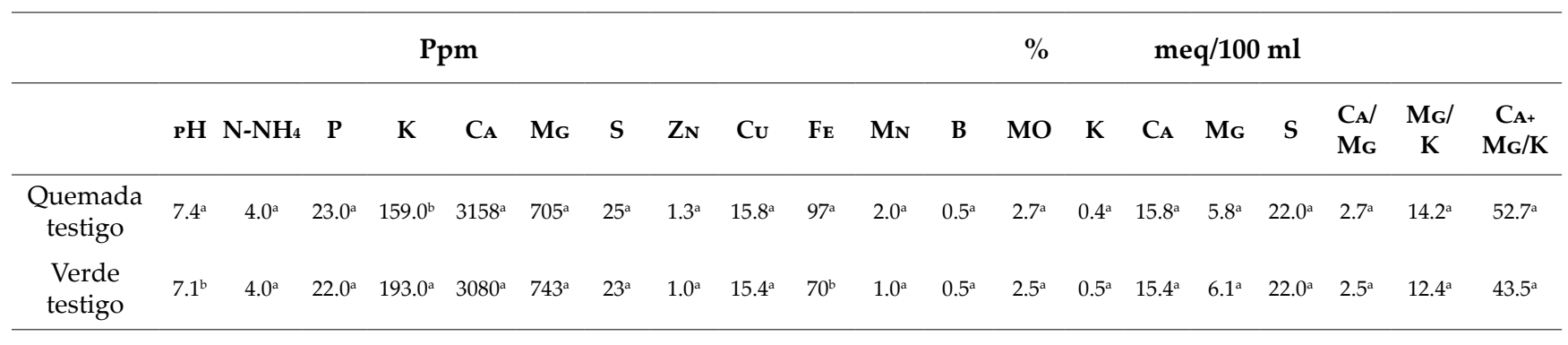

z: letras distintas en el sentido de las columnas indican diferencias significativas, de acuerdo con la prueba $t-S t u d e n t(p=0.05)$.

quemada durante los dos primeros meses posteriores a la cosecha. Esto representa $18.51 \%$ restituyéndose posteriormente al valor de partida, lo cual podría ser un error de laboratorio o contaminación de la muestra, ya que es muy poco probable que en un mes se incremente $0.4 \%$ de $\mathrm{MO}$ en condiciones de caña quemada y sin riego. La caña verde se mantuvo estable con tendencia al incremento, lo que coincide con lo encontrado por Wiedenfeld (2009): un aumento en el contenido de materia orgánica después de tres años de evaluaciones en condiciones similares. Otro aspecto importante es el planteado por Souza et al. (2012) y Loss et al. (2009), quienes refieren que la quema proporciona mayor disponibilidad de carbono mineralizable y de fácil descomposición, mientras que la no quema favorece la fracción recalcitrante, lo que conlleva a una menor perdida de carbono y, por tanto, favorece las propiedades físico-químicas de los suelos.

Esto puede explicar por qué el contenido de nitrógeno amoniacal se incrementó levemente en la caña quemada, lo que no coincide con Souza et al. (2012), quienes manifiestan que al haber mayor contenido de materia orgánica del suelo, abundan las formas orgánicas del nitrógeno. Sin embargo, atendiendo que aumenta la mineralización por la oxidación de la materia orgánica debido a la quema de la caña, podría inferirse que en las primeras etapas debe existir incremento del contenido del nitrógeno en la tecnología quemada que después, producto del proceso de nitrificación, disminuiría por el lavado de nitratos (NO3-) (Groffman y Rosi-Marshall 2013). La tasa de mineralización de los residuos de la caña de azúcar verde es lenta por la alta concentración de lignina que poseen (Chen 2014). Esto provoca que el aporte de nitrógeno orgánico sea bajo en comparación con la quema en primera instancia, que posteriormente se incrementa por mantener mayor humedad $\mathrm{y}$ actividad biológica (explicado más adelante).
El contenido de fósforo tuvo una disminución al inicio de quemarse la caña de azúcar que fue incrementando con el paso del tiempo, mientras que en la caña verde siempre se mantuvo en cantidades homogéneas, lo que coincide con lo planteado por Pavinato y Rosolem (2008) quienes refieren que el contenido de este elemento se eleva por el incremento de residuos vegetales. Precisamente la disminución drástica de los residuos vegetales con la quema puede originar un detrimento del contenido de fósforo, que se restituirá con la aparición paulatina de materia orgánica nueva; además, una disminución del $\mathrm{pH}$, acercándolo a neutro, influye en que aumente el contenido de fosforo disponible porque disminuyen los fosfatos de calcio (Navarro y Navarro 2013).

Los niveles de potasio se mantuvieron estables en caña verde mientras que en la quemada se evidencia una disminución significativa, lo cual coincide con los estudios de Souza et al. (2012) y Canellas et al. (2002), quienes encontraron un aumento de potasio en la caña sin quemar respecto a la quemada. Una explicación probable parece ser que la textura arcillosa del suelo y el tipo de arcilla (2:1) favorece el proceso de fijación del potasio, que ocurre cuando el catión potasio queda retenido dentro de la estructura cristalina de este tipo de arcillas. Esto se agudiza cuando disminuye la humedad; sin embargo, en el sistema de cosecha en verde al mantener una mayor humedad las arcillas parecen permanecer más tiempo expandidas, por lo que el potasio queda más disponible (Raheb y Heidari 2012).

El contenido de magnesio, azufre, zinc y cobre tuvieron un incremento en los primeros meses en la caña quemada, que fue disminuyendo a medida que transcurrió el tiempo. En caña verde, por su parte, se mantuvieron estables o se incrementaron establemente; el calcio y el boro se registraron en cantidades homogéneas en ambos sistemas. 
Cuadro 3. Resultados analíticos del primer mes posterior a la cosecha de caña de azúcar bajo dos sistemas de producción en Cantón Milagro, Ecuador.

\begin{tabular}{|c|c|c|c|c|c|c|c|c|c|c|c|c|c|c|c|c|c|c|c|c|}
\hline & & & & & & Ppm & & & & & & & $\%$ & & me & $q / 100$ & $\mathrm{ml}$ & & & \\
\hline & $\mathbf{p H}$ & $\mathrm{N}-\mathrm{NH}_{4}$ & $\mathbf{P}$ & $\mathbf{K}$ & $\mathrm{C}_{\mathbf{A}}$ & Mg & $S$ & $\mathrm{Zn}$ & $\mathrm{Cu}$ & Fe & MN & B & MO & $\mathbf{K}$ & $\mathrm{C}_{\mathbf{A}}$ & Mg & $S$ & $\begin{array}{l}\text { CA/ } \\
\text { MG }\end{array}$ & $\begin{array}{c}\text { MG/ } \\
\text { K }\end{array}$ & $\begin{array}{c}\mathrm{CA}_{\mathrm{A}+} \\
\mathrm{MG} / \mathrm{K}\end{array}$ \\
\hline Quemada & $7.2^{\mathrm{a}}$ & $3.0^{\mathrm{a}}$ & $18.0^{\mathrm{b}}$ & $134.0^{\mathrm{b}}$ & $3216.0^{\mathrm{a}}$ & $770.0^{\mathrm{a}}$ & $28.0^{\mathrm{a}}$ & $3.4^{\mathrm{a}}$ & $16.4^{a}$ & $58.0^{\mathrm{a}}$ & $4.0^{\mathrm{a}}$ & $0.7^{\mathrm{a}}$ & $2.2^{\mathrm{b}}$ & $0.3^{\mathrm{b}}$ & $16.1^{\mathrm{a}}$ & $6.3^{\mathrm{a}}$ & $22.8^{\mathrm{a}}$ & $2.5^{\mathrm{a}}$ & $18.4^{\mathrm{a}}$ & $65.2^{\mathrm{a}}$ \\
\hline Verde & $6.9^{\mathrm{b}}$ & $3.0^{\mathrm{a}}$ & $21.0^{\mathrm{a}}$ & $186.0^{\mathrm{a}}$ & $3263.0^{\mathrm{a}}$ & $749.0^{\mathrm{a}}$ & $24.0^{\mathrm{a}}$ & $1.2^{\mathrm{b}}$ & $15.2^{\mathrm{a}}$ & $60.0^{\mathrm{a}}$ & $2.0^{\mathrm{a}}$ & $0.9^{a}$ & $2.5^{\mathrm{a}}$ & $0.5^{\mathrm{a}}$ & $16.3^{\mathrm{a}}$ & $6.2^{\mathrm{a}}$ & $22.9^{\mathrm{a}}$ & $2.7^{\mathrm{a}}$ & $12.9^{\mathrm{b}}$ & $47.1^{\mathrm{b}}$ \\
\hline
\end{tabular}

z: letras distintas en el sentido de las columnas indican diferencias significativas, de acuerdo con la prueba $t$-Student $(p=0.05)$.

Cuadro 4. Resultados analíticos del segundo mes posterior a la cosecha de caña de azúcar bajo dos sistemas de producción en Cantón Milagro, Ecuador.

\begin{tabular}{|c|c|c|c|c|c|c|c|c|c|c|c|c|c|c|c|c|c|c|c|c|}
\hline & & & & & & Ppm & & & & & & & $\%$ & & me & $\mathrm{q} / \mathbf{1 0 0}$ & ml & & & \\
\hline & $\mathbf{p H}$ & $\mathrm{N}-\mathrm{NH}_{4}$ & $\mathbf{P}$ & $\mathbf{K}$ & $\mathrm{CA}_{\mathrm{A}}$ & Mg & $S$ & $\mathrm{Zn}$ & $\mathrm{Cu}$ & Fe & MN & B & MO & $\mathbf{K}$ & $\mathrm{CA}_{\mathrm{A}}$ & Mg & $S$ & $\begin{array}{l}\mathrm{CA}_{\mathbf{A}} / \\
\mathrm{MG}\end{array}$ & $\begin{array}{c}\text { MG/ } \\
\text { K }\end{array}$ & $\begin{array}{c}\mathrm{C}_{\mathbf{A}+} \\
\mathrm{MG}_{\mathbf{G}} / \mathrm{K}\end{array}$ \\
\hline Quemada & $7.2^{\mathrm{a}}$ & $4.0^{\mathrm{a}}$ & $18.0^{\mathrm{b}}$ & $131.0^{\mathrm{b}}$ & $3313.0^{\mathrm{a}}$ & $781.0^{\mathrm{a}}$ & $25.0^{\mathrm{a}}$ & $1.4^{\mathrm{a}}$ & $14.9^{\mathrm{a}}$ & $54.0^{\mathrm{b}}$ & $4.0^{\mathrm{a}}$ & $0.8^{\mathrm{a}}$ & $2.2^{\mathrm{b}}$ & $0.3^{\mathrm{b}}$ & $16.6^{\mathrm{a}}$ & $6.4^{\mathrm{a}}$ & $23.3^{\mathrm{a}}$ & $2.6^{\mathrm{a}}$ & $19.1^{\mathrm{a}}$ & $68.5^{\mathrm{a}}$ \\
\hline Verde & $7.2^{\mathrm{a}}$ & $3.0^{\mathrm{a}}$ & $23.0^{\mathrm{a}}$ & $181.0^{\mathrm{a}}$ & $3329.0^{\mathrm{a}}$ & $727.0^{\mathrm{b}}$ & $27.0^{\mathrm{a}}$ & $1.9^{\mathrm{a}}$ & $15.4^{\mathrm{a}}$ & $92.0^{\mathrm{a}}$ & $1.0^{\mathrm{b}}$ & $0.8^{\mathrm{a}}$ & $2.5^{\mathrm{a}}$ & $0.5^{\mathrm{a}}$ & $16.7^{\mathrm{a}}$ & $6.0^{\mathrm{b}}$ & $23.1^{\mathrm{a}}$ & $2.8^{\mathrm{a}}$ & $12.9^{\mathrm{b}}$ & $48.8^{\mathrm{b}}$ \\
\hline
\end{tabular}

z: letras distintas en el sentido de las columnas indican diferencias significativas, de acuerdo con la prueba $t$-Student $(p=0.05)$.

Cuadro 5. Resultados analíticos del tercer mes posterior a la cosecha de caña de azúcar bajo dos sistemas de producción en Cantón Milagro, Ecuador.

\begin{tabular}{|c|c|c|c|c|c|c|c|c|c|c|c|c|c|c|c|c|c|c|c|c|}
\hline & & & & & & Ppm & & & & & & & $\%$ & & me & $q / 100$ & $\mathrm{ml}$ & & & \\
\hline & $\mathbf{P H}$ & $\mathrm{N}-\mathrm{NH}_{4}$ & $\mathbf{P}$ & $\mathbf{K}$ & $\mathrm{C}_{\mathbf{A}}$ & $\mathrm{Mg}$ & $S$ & $\mathrm{Zn}$ & $\mathrm{Cu}$ & FE & $\mathbf{M N}_{\mathbf{N}}$ & B & MO & $\mathbf{K}$ & $\mathrm{C}_{\mathbf{A}}$ & Mg & $S$ & $\begin{array}{l}\mathrm{C}_{\mathbf{A}} / \\
\mathrm{MG}\end{array}$ & $\begin{array}{c}\text { MG/ } \\
\text { K }\end{array}$ & $\begin{array}{c}\mathrm{CA}_{\mathbf{A}+} \\
\mathbf{M G} / \mathrm{K}\end{array}$ \\
\hline Quemada & $7.1^{\mathrm{a}}$ & $4.6^{\mathrm{a}}$ & $21.3^{\mathrm{a}}$ & $142.3^{\mathrm{b}}$ & $3329.0^{\mathrm{a}}$ & $726.0^{\mathrm{a}}$ & $27.0^{\mathrm{a}}$ & $1.0^{\mathrm{a}}$ & $13.9^{\mathrm{a}}$ & $73.3^{\mathrm{a}}$ & $4.7^{\mathrm{a}}$ & $0.8^{\mathrm{a}}$ & $2.6^{\mathrm{a}}$ & $0.4^{\mathrm{a}}$ & $16.5^{\mathrm{a}}$ & $6.0^{\mathrm{a}}$ & $23.0^{\mathrm{a}}$ & $2.8^{\mathrm{a}}$ & $16.9^{\mathrm{a}}$ & $64.0^{\mathrm{a}}$ \\
\hline Verde & $6.9^{\mathrm{a}}$ & $4.3^{\mathrm{a}}$ & $22.7^{\mathrm{a}}$ & $188.0^{\mathrm{a}}$ & $3167.7^{b}$ & b $733.0^{a}$ & $28.0^{\mathrm{a}}$ & $1.8^{\mathrm{a}}$ & $15.0^{\mathrm{a}}$ & $77.0^{\mathrm{a}}$ & $4.0^{\mathrm{a}}$ & $1.0^{\mathrm{a}}$ & $2.6^{\mathrm{a}}$ & $0.5^{\mathrm{a}}$ & $15.8^{\mathrm{a}}$ & $6.0^{\mathrm{a}}$ & $22.4^{\mathrm{a}}$ & $2.6^{\mathrm{a}}$ & $12.7^{\mathrm{b}}$ & $45.8^{\mathrm{b}}$ \\
\hline
\end{tabular}

z: letras distintas en el sentido de las columnas indican diferencias significativas, de acuerdo con la prueba $t$-Student ( $p=0.05$ ).

El hierro tuvo una disminución significativa en caña quemada que fue recuperando paulatinamente en el transcurso del tiempo; el manganeso tuvo un incremento significativo en ambos sistemas, que resultó mayor en la verde.

En la interacción de nutrientes, se puede observar que en la caña quemada existe antagonismo bien marcado del calcio y el magnesio sobre el potasio (en Ecuador las relaciones aceptadas son $\mathrm{Mg} / \mathrm{K}: 2.5$ - 10 y $(\mathrm{Ca}+\mathrm{Mg}) / \mathrm{K}: 12.5$ - 50, según las recomendaciones del Laboratorio de Suelos, Tejidos Vegetales y Agua del Instituto Nacional de Investigaciones Agropecuarias), lo que puede provocar que disminuya la dis- ponibilidad de este elemento para las plantas (Engels et al. 2012) y por consiguiente potencial disminución del rendimiento (variable no considerada en este estudio), ya que este elemento es el de mayor consumo (Humbert 2013).

\section{Agregados estables del suelo o índice estructural}

En la figura 1 se evidencia un efecto marcado sobre la estabilidad estructural del suelo: la quema de caña disminuye este índice llevándola a la categoría más baja (suelos con alta susceptibilidad a la erosión y a la degradación física). Con la tecnología verde, por 


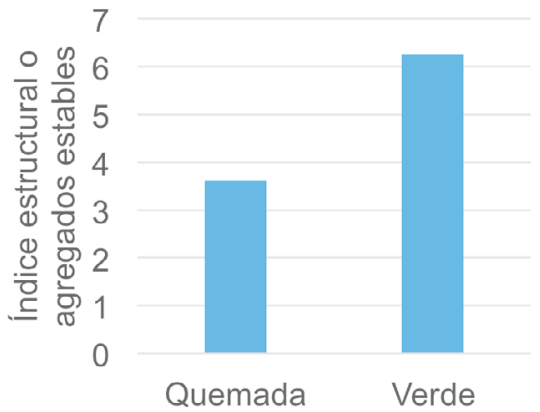

Figura 1. Efecto de la cosecha caña verde y caña quemada sobre la estabilidad estructural del suelo en Cantón Milagro, Ecuador.

su parte, la estabilidad estructural se mantuvo en la categoría superior, según las categorías de Pieri (1995).

Esto coincide con lo reportado por Blair (2000), quien, al comparar la cosecha verde con la quemada, comprobó mediante otra metodología (inmersión en húmedo) que la tecnología verde logró mantener mayor estabilidad estructural. Esto provoca que los suelos resistan más la acción disgregante del agua y los procesos erosivos que conllevan a un aumento de su resiliencia y posibilidad de mantener una mejor estructura.

\section{Actividad biológica}

En la figura 2 se puede observar un ligero incremento $(4.6 \%)$ de la actividad biológica a las cuatro semanas, mientras que a las 12 semanas ésta superó en 16.8\% a la de la cosecha quemada, aspecto que coincide con el reportado por Rachid et al. (2013), quienes manifiestan que la quema disminuye la diversidad y el número de colonias de los microorganismos del suelo.

Una mayor actividad biológica, provee una mayor mineralización de la materia orgánica y aceleración de los ciclos biogeoquímicos, lo que hace más disponible los elementos nutritivos a las plantas. Con base a lo anterior, Tortora et al. (2013) plantean que los residuos de caña pueden incrementar las colonias de levaduras, hongos y poblaciones de Pseudomonas sp., especialmente en climas cálidos. Los microorganismos aislados en ese estudio tuvieron actividad lignolítica y solubilizadores de fosforo, especialmente géneros de Pseudomonas; también hubo un incremento de bacterias fijadoras de nitrógeno. Esto puede explicar, en parte, que la fertilidad se mantuviese ligeramente superior en la cosecha verde en relación con la quemada.

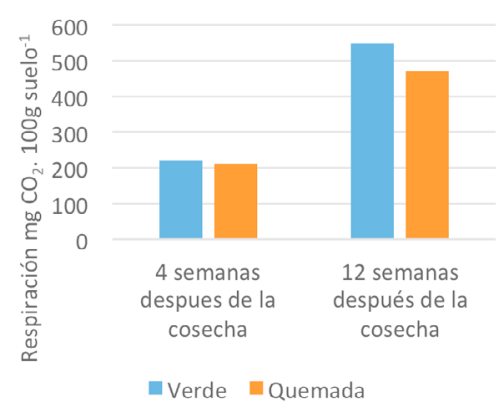

Figura 2. Efecto de la cosecha caña verde y caña quemada sobre la actividad biológica del suelo en Cantón Milagro, Ecuador.

\section{Incremento de la humedad}

Se evidenció un incremento de la humedad (figura 3) de $48 \%$ en el caso de la tecnología de cosecha verde a diferencia de la cosecha quemada; ésta es una de las principales ventajas de la tecnología de cosecha verde para la conservación del suelo. Lo anterior coincide parcialmente con lo reportado por Núñez y Spaans (2008), quienes manifiestan que hubo un incremento de sólo $2 \%$ del contenido de agua del suelo. Esto representó una disminución significativa de la evapotranspiración que permite reducir los ciclos de irrigación del cultivo y, por ende, los costos de producción por este concepto.

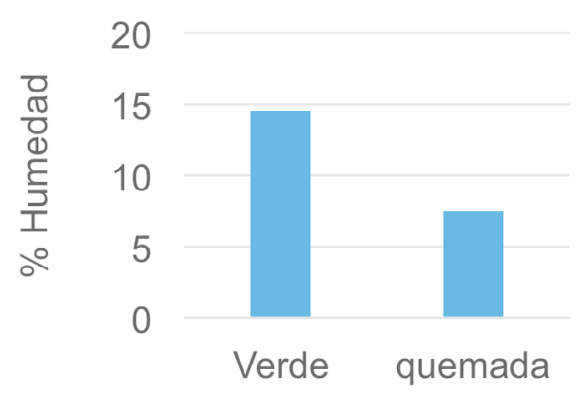

Figura 3. Efecto de la cosecha caña verde y cosecha quemada sobre la humedad (\%) del suelo en Cantón Milagro, Ecuador.

Según Alamilla-Magaña et al. (2016), al disponer de un mayor contenido de humedad y contenido de agua a menor tensión, la planta logra alcanzar un mayor rendimiento y cantidad de sacarosa con respecto a las plantas con déficit hídrico, factores que pueden disminuir notablemente el crecimiento 


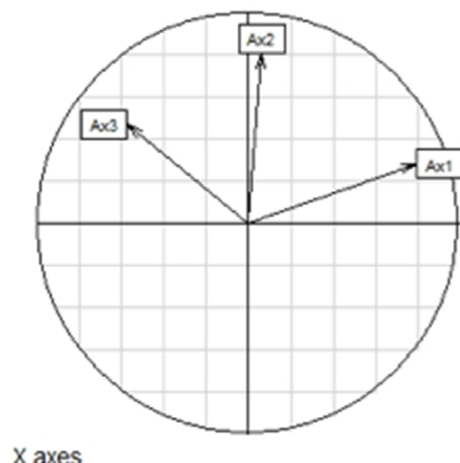

$\mathrm{X}$ axes

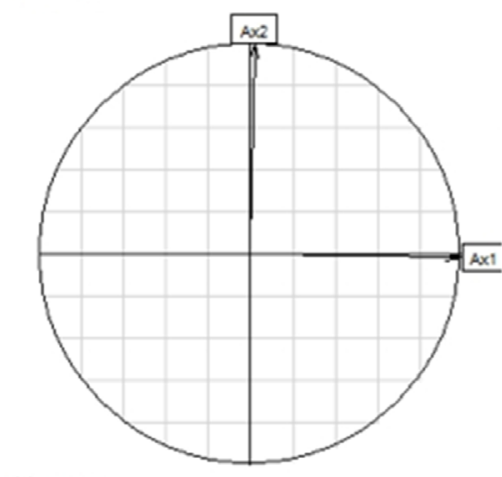

$Y$ axes

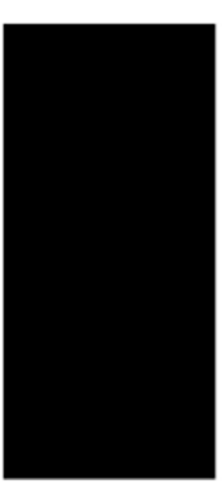

Eigenvalues

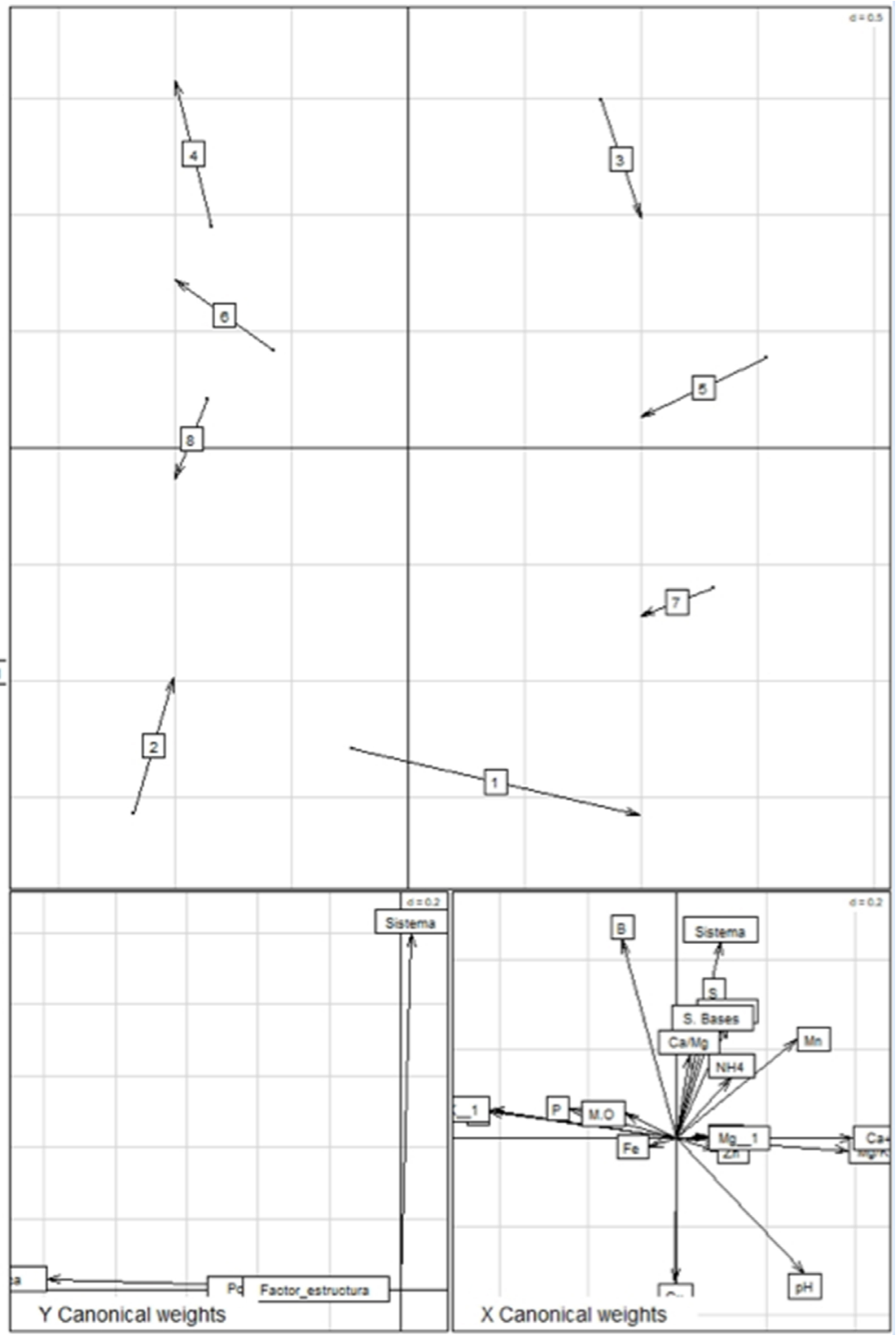

1. caña verde testigo; 2 . caña quemada testigo; 3 . caña verde 1 mes; 4 . caña quemada 1 mes; 5 . caña verde 2 meses; 6 . caña quemada 2 meses; 7 . caña verde tres meses, y 8. caña quemada tres meses.

Figura 4. Resultados del análisis de CoInercia entre variables uso de suelo y propiedades edáficas: a) plano factorial de CoInercia de los usos de suelo; b) proyección de los vectores de factores físicos - biológicos, y c) de las variables químicas del suelo en el plano factorial de CoInercia.

y grosor del tallo, sin que afecte su concentración de sacarosa, ni las principales propiedades aceptadas por la industria. Por ello, el uso de la cosecha verde potenciaría un incremento de la producción y una disminución de los gastos destinados al riego, sin contar que existen muchas zonas que no están irrigadas, donde el efecto sería mucho mayor debido a la imposibilidad de restituir el agua pérdida.

En el análisis de Coinercia (figura 4) se observa que la caña verde mostró mejor relación con las principales propiedades del suelo, lo cual se evidencia en que todas las evaluaciones de caña verde están en el plano factorial y correlacionadas con la mayoría de las propiedades evaluadas, sin embargo la caña quemada tuvo una mayor relación con el contenido de $\mathrm{P}$ y de materia orgánica, lo que indica que desde el inicio, después de cortada la caña, de manera general la tecnología verde resultó tener un mejor efecto o menor impacto, desde el punto de vista de la conservación y el mantenimiento de la fertilidad del suelo. 


\section{Conclusiones}

No existió diferencia significativa entre la cosecha verde y la quemada con respecto a la fertilidad química del suelo; solamente fueron afectados el contenido y bloqueo de potasio, el $\mathrm{pH}$ y el contenido de materia orgánica. En el sistema de cosecha verde se evidencia un incremento de la actividad biológica del suelo. Con el suelo cubierto por los desechos de la cosecha de caña de azúcar se mantiene la humedad hasta $48 \%$ más que cuando está desnudo. La tecnología de caña quemada tiene un efecto negativo sobre la estabilidad estructural del suelo, lo que puede acelerar el proceso de degradación. El sistema de cosecha de caña verde constituye la tecnología más adecuada desde el punto de vista edáfico ya que tuvo un mejor comportamiento sobre las propiedades físicas, químicas y biológicas evaluadas.

\section{Agradecimientos}

Se agradece a la Universidad Agraria de Ecuador por la financiación del Proyecto "Efecto de dos sistemas de manejo de la caña de azúcar sobre la calidad del suelo y diseño de estrategias para el mejoramiento y conservación del medio edáfico", con Resolución del Honorable Consejo Universitario No. 182-2015.

\section{LiTERATURA CITADA}

Alamilla-Magaña JC, Carrillo-Ávila E, Obrador-Olán JJ, Landeros-Sánchez C, Vera-Lopez J, Juárez-López JF. 2016. Soil moisture tension effect on sugar cane growth and yield. Agricultural Water Management 177: 264273. https://doi.org/10.1016/j.agwat.2016.08.004

Arbex MA, Martins LC, de Oliveira RC, Pereira LAA, Arbex FF, Cançado JED, Saldiva PHN, Braga ALF. 2007. Air pollution from biomass burning and asthma hospital admissions in a sugar cane plantation area in Brazil. Journal of Epidemiology \& Community Health 61(5): 395-400. https://doi.org/10.1136/jech.2005.044743

Blair N. 2000. Impact of cultivation and sugar-cane green trash management on carbon fractions and aggregate stability for a Chromic Luvisol in Queensland, Australia. Soil and Tillage Research 55(3): 183-191. https://doi. org/10.1016/S0167-1987(00)00113-6
Canellas LP, Velloso ACX, Marciano CR, Ramalho JFGP, Rumjanek VM, Rezende CE, Santos GA. 2003. Chemical soil properties of an inceptisol under long-term sugarcane crops with vinasse application and without slash burning. Revista Brasileira de Ciência do Solo 27(5): 935944. https://doi.org/10.1590/S0100-06832003000500018

Chaves M, Bermúdez AZ. 2006. Motivos y razones para quemar las plantaciones de caña de azúcar en Costa Rica. Memoria "XVI Congreso ATACA y XVI Congreso ATACORI". Heredia, Costa Rica. p: 248-253.

Chen H. 2014. Biological fundamentals for the biotechnology of lignocellulose. In: Chen H, editor. Biotechnology of lignocellulose. Dordrecht, Springer. p: 73-141.

[CINCAE] Centro de Investigación de la Caña de Azúcar del Ecuador. [internet]. 2017. Informe anual 2016. Acceso: 20 de noviembre de 2017. Disponible en: 
http://cincae.org/wp-content/uploads/2013/04/Informe-Anual-2016.pdf

Dray S, Dufour A-B. 2007. The ade4 Package: implementing the duality diagram for ecologists. Journal of Statistical Software 22(4): 1-20.

Engels C, Kirkby E, White P. 2012. Mineral nutrition, yield and source-sink relationships. In: Marschner P, editor. Marschner's mineral nutrition of higher plants. San Diego, Academic Press. p: 85-133.

Groffman PM, Rosi-Marshall EJ. 2013. Chapter 7 - The Nitrogen Cycle. Weathers KC, Strayer DL, Likens GE, editor. Fundamentals of ecosystem science. Amsterdam, Academic Press. p: 137-158.

Humbert RP. 2013. The nutrition of sugar cane. In: Humbert RP, editor. The growing of sugar cane. Amsterdam, Elsevier. p: 114-301.

IBM CORP. Released. 2013. IBM SPSS Statistics for Windows, Version 22.0. IBM Corp. Armonk, USA.

Isermeyer H. 1952. Eine einfache methode zur bestimmung der bodenatmung und der karbonate im boden. Zeitschrift für Pflanzenernährung, Düngung, Bodenkunde 56: 26-38. https://doi.org/10.1002/jpln.19520560107

Loss A, Pereira MG, Ferreira EP, dos Santos LL, Beutler SJ, Ferraz Júnior ASL. 2009. Frações oxidáveis do carbono ornânico em argissolo vermelho-amarelo sob sistema de aleias. Revista Brasileira de Ciência do Solo 33: 867874. https://doi.org/10.1590/S0100-06832009000400011

Mendoza HNS, Lima E, Anjos LHC, Silva LA, Ceddia MB, Antunes MVM. 2000. Propriedades químicas e biológicas de solo de tabuleiro cultivado com cana-de-açúcar com e sem queima da palhada. Revista Brasileira de Ciência do Solo 24: 201-207. https://doi.org/10.1590/ S0100-06832000000100022

Navarro G, Navarro S. 2013. Química agrícola: química del suelo y de los nutrientes esenciales para las plantas. Mundi-Prensa. Madrid, España.

Núñez O, Spaans E. 2008. Evaluation of green-cane harvesting and crop management with a trash-blanket. Sugar Tech 10(1): 29-35. https://doi.org/10.1007/s12355-0080005-1

Ortiz H, Salgado S, Castelán M, Córdova S. 2012. Perspectivas de la cosecha de la caña de azúcar cruda en México. Revista Mexicana de Ciencias Agrícolas Publicación Especial 4: 767-773.

Pavinato PS, Rosolem CA. 2008. Disponibilidade de nutrientes no solo - decomposição e liberação de compostos orgânicos de resíduos vegetais. Revista Brasileira de Ciência do Solo 32(3): 911-920. https://doi.org/10.1590/ S0100-06832008000300001

Pieri C. 1995. Long-term soil management experiments in semiarid francophone Africa. In: Lal R, Steward BA, editors. Soil management: experimental basis for sustainability and environmental quality. Boca Raton, CRC. p: 225-266.
Pongpat P, Gheewala SH, Silalertruksa T. 2017. An assessment of harvesting practices of sugarcane in the central region of Thailand. Journal of Cleaner Production 142(3): 1138-1147. https://doi.org/10.1016/j.jclepro.2016.07.178

R Core Team. [internet]. 2017. R: A language and environment for statistical computing. R Foundation for Statistical Computing. Descargado: Dicembre de 2017. Disponible en: https://www.R-project.org/

Rachid CTCC, Santos AL, Piccolo MC, Balieiro FC, Coutinho HLC, Peixoto RS, Tiedje JM, Rosado AS. 2013. Effect of sugarcane burning or green harvest methods on the Brazilian Cerrado soil bacterial community structure. PLoS ONE 8(3): e59342. https://doi.org/10.1371/journal. pone.0059342

Raheb A, Heidari A. 2012. Effects of clay mineralogy and physico-chemical properties on potassium availability under soil aquic conditions. Journal of Soil Science and Plant Nutrition 12(4): 747-761. https://doi.org/10.4067/ S0718-95162012005000029

Souza RA, Telles TS, Machado W, Hungria M, Filho JT, Guimarães MDF. 2012. Effects of sugarcane harvesting with burning on the chemical and microbiological properties of the soil. Agriculture, Ecosystems \& Environment 155: 1-6. https://doi.org/10.1016/j.agee.2012.03.012

Tortora ML, Vera L, Grellet N, Fernández J, Digonzelli PA, Romero RR. 2013. Effect of sugar cane trash blanketing on the development of microorganisms of agronomic and environmental interest. Revista Industrial y Agrícola de Tucumán 90(1): 61-68.

[USDA] Departamento de Agricultura de los Estados Unidos. 2014. Claves para la taxonomía de suelos. Washington, USA.

Wiedenfeld B. 2009. Effects of green harvesting vs burning on soil properties, growth and yield of sugarcane in South Texas. Journal of the American Society of Sugar Cane Technologists 29: 102-109. 